\title{
Clozapine Is Better Tolerated in Younger Patients: Risk Factors for Discontinuation from a Nationwide Database in Japan
}

\author{
Katsunori Toyoda', Takeo Hata ${ }^{2}$, Shigeru Yamauchi', Shinya Kinoshita', \\ Masami Nishihara ${ }^{2}$, Kazuhisa Uchiyama ${ }^{2}$, Ken Inada ${ }^{3}$, and Tetsufumi Kanazawa ${ }^{1}$ \\ 1'Department of Neuropsychiatry, Osaka Medical College, Osaka, Japan \\ ${ }^{2}$ Department of Pharmacy, Osaka Medical College Hospital, Osaka, Japan \\ ${ }^{3}$ Department of Psychiatry, Tokyo Women's Medical University, Tokyo, Japan
}

\begin{abstract}
Objective The effectiveness of clozapine is clearly superior to other antipsychotics in the treatment of refractory schizophrenia. Clozapine leads to various side effects, and therefore many patients are forced to discontinue. In this study, we analyzed the registry database of all cases in Japan to identify risk factors for discontinuation of clozapine.

Methods The Clozaril patient monitoring service ${ }^{\circledR}$ (CPMS) database from July 31, 2009 to January 26, 2020 was acquired. We defined the following exclusion criteria: patients who had ever taken clozapine by a non-CPMS method, such as an individual import or clinical trial, patients who did not receive clozapine after being enrolled in CPMS, and patients with initial doses other than $12.5 \mathrm{mg}$ (outside the current protocol). Therefore, all patients in this study are new users. Multivariate Cox regression analysis was used to investigate independent risk factors associated with time to discontinuation of clozapine.

Results We identified 8,263 patients as the study population. Clozapine discontinuation was significantly associated with age 40 and older [hazard ratio $(\mathrm{HR})=1.66, \mathrm{p}<0.001$ ], intolerance to olanzapine $(\mathrm{HR}=1.31, \mathrm{p}=0.018)$, previous treatment with clozapine ( $\mathrm{HR}=1.30$, $\mathrm{p}=0.001)$, and leukocyte counts $<6,000 / \mathrm{mm}^{3}(\mathrm{HR}=1.24, \mathrm{p}<0.001)$. The Kaplan-Meier curves for clozapine discontinuation by age group revealed that older age at the time of clozapine introduction tended to have lower continuation rates.

Conclusion Careful administration is important because patients with these factors have a high risk of discontinuation. In addition, the initiation of clozapine during the younger period was more effective and more tolerated. Psychiatry Investig 2021;18(2):101-109
\end{abstract}

Key Words Antipsychotics, Clozapine, Continuation rate, Leukopenia, Olanzapine, Treatment-resistant schizophrenia.

\section{INTRODUCTION}

Schizophrenia continues to be an important disease in psychiatry. Schizophrenia is consistently high in terms of disability-adjusted life years, ${ }^{1}$ the resources lost to caregivers such as family members, ${ }^{2}$ the high suicide rate, ${ }^{3}$ and the economic cost for society as a whole. ${ }^{4}$ In the case of treatment-resistant schizophrenia, these numbers become higher. Individuals with treatment-resistant schizophrenia are often nonresponsive even with sufficient drug treatment and/or a group whose tolera-

Received: October 13, 2020 Revised: November 3, 2020 Accepted: November 11, 2020

$\triangle$ Correspondence: Tetsufumi Kanazawa, MD, PhD

Department of Neuropsychiatry, Osaka Medical College, 2-7 Daigaku-machi, Takatsuki, Osaka 569-8686, Japan

Tel: +81-72-683-1221, E-mail: psy052@osaka-med.ac.jp

(c) This is an Open Access article distributed under the terms of the Creative Commons Attribution Non-Commercial License (https://creativecommons.org/licenses/bync/4.0) which permits unrestricted non-commercial use, distribution, and reproduction in any medium, provided the original work is properly cited. bility of drug treatment could not be maintained due to side effects such as extrapyramidal symptoms. ${ }^{5}$

The most effective treatment for refractory schizophrenia is continuous administration of clozapine. ${ }^{6}$ The efficacy of clozapine compared to other antipsychotic treatments has been revealed by various indicators, reduction in mortality rate including suicide, improvement rate based on psychotic symptoms, ${ }^{8}$ reduction in readmissions and recurrences, ${ }^{9}$ and reduction in medical costs. ${ }^{10}$ In Japan, clozapine was introduced in 2009 , although the number of prescriptions for clozapine is still low. The age-adjusted use ratio is 0.9 per $100,000,{ }^{11}$ which is the lowest rate of clozapine use among 15 Asian countries surveyed in 2016. ${ }^{12}$ Although clozapine has a better prognosis for patients who continue as long as possible, ${ }^{13}$ there are many patients who have to be discontinued due to serious side effects such as myocarditis and agranulocytosis. ${ }^{14}$ Given the role of clozapine as the last resort in treatment-resistant schizophrenia, it is important to obtain information on risk factors for clo- 
zapine discontinuation and when and how more effective clozapine therapy should be introduced. Information such as whether the previous antipsychotic drug contributes to a high continuation rate of clozapine is useful not only for individuals with treatment-resistant schizophrenia but also for efficient medical resource allocation.

A small study previously reported that in African-Americans (in a study on racial differences), ${ }^{15}$ the introduction of clozapine at a high age and the coexistence of substance abuse are risk factors for clozapine discontinuation. ${ }^{16}$ Although a UK Clozaril patient monitoring service ${ }^{\circledR}$ (CPMS) database study in 1999 reported high discontinuation rates in the early stages of treatment and a high incidence of agranulocytosis in those with Asian ethnicity, ${ }^{17}$ a predictable high risk factor for clozapine discontinuation has not been identified. Therefore, in the current study, we conducted an analysis using a registry database of all cases in Japan to identify the risk factors that would be discontinued after the initiation of clozapine in a sufficient sample size, and the information should improve the therapeutic effect.

\section{METHODS}

\section{The definition of treatment-resistant schizophrenia and the regulation of clozapine treatment in Japan}

Treatment-resistant schizophrenia, which is indicated for clozapine in Japan, is defined by two criteria. The first is poor response, with limited response after an administration of sufficient doses of two or more antipsychotics for 4 weeks or longer. It is at least $600 \mathrm{mg} /$ day of chlorpromazine equivalent and contains one or more second-generation antipsychotics. The second is poor tolerability, the dose of antipsychotics could not be increased sufficiently due to side effects. In Japan, clozapine induction is performed in the hospital, and it requires inpatient treatment up to 18 weeks after the administration. Regular blood test at least once a week up to 26 weeks is also demanded. After 26 weeks, it is needed follow-up with a blood test at least once every two weeks.

\section{Data source}

Data were obtained from the CPMS center as requested by the Japanese Society of Neuropsychopharmacology. The CPMS data consisted of registered data for all patients who had received clozapine in Japan between July 31, 2009 and January 26, 2020. The CPMS data we acquired were anonymized, and each patient was given a unique identification code, which included patient registration date, registered medical institution, sex, date of birth, test date, leukocyte count, neutrophil count, blood glucose level, hemoglobin A1c (HbA1c), and prescription date. If a patient had previously discontinued clozapine for any reason and then readministered clozapine, the patient was newly enrolled and treated as a separate case. Due to personal information protection considerations, patient consent requirements were waived.

\section{Exclusion criteria}

To describe the risk factors for clozapine discontinuation using the CPMS database, we defined the following exclusion criteria: patients who have ever taken clozapine by a non-CPMS method, such as an individual import or clinical trial, patients who did not receive clozapine after being enrolled in CPMS, and patients with initial doses other than $12.5 \mathrm{mg}$ (outside the current protocol). Therefore, all patients in this study were new users with clozapine administration that was compatible with the standard protocol.

\section{Definition of terms}

Patient age was defined as the age at the first prescription of clozapine. The clozapine treatment period was defined as the period from the first prescription date to the last prescription date plus the number of prescription days. Patients who did not register their records in CPMS for discontinuation of clozapine by the observation end date, January 26, 2020, were clozapine continued cases, that is, it was called a clozapine censored case. Neutropenia and leukopenia were defined as medical conditions with neutrophil counts of less than $1,500 / \mathrm{mm}^{3}$ and leukocyte counts of less than $3,000 / \mathrm{mm}^{3}$, respectively. In Japan, if the laboratory values fell into these ranges, the clozapine prescription must be immediately suspended according to CPMS criteria. Furthermore, agranulocytosis was defined as a neutrophil count of less than $500 / \mathrm{mm}^{3}$.

\section{Statistical analysis}

The Kaplan-Meier event-free curve was used to calculate the event-free probability of clozapine discontinuation. A logrank test was performed to verify the differences between each group. Multivariate Cox regression analysis was used to investigate independent risk factors associated with time to event. The event was the discontinuation of clozapine. Variance inflation factors (VIFs) of 10 or more were considered evidence of the existence of multicollinearity. The proportional hazards assumption was evaluated using the Kaplan-Meier curve. A trend test for survival data was performed for trends by age group in time to event. For the trend test, we assigned the median age within each level in the age group as the variable, that is, 18 for " $\leq 20$," 27 for “ $21-30$," 36 for “ $31-40$," 45 for “ $41-50$," 55 for “51-60," 64 for “61-70," and 73 for “ $\geq 71$." A Cox regression model was fitted to assess the trend of age group assigned these values. The Cochran-Armitage trend test was performed to clarify the trend of incidence of neutropenia/leukopenia and 
agranulocytosis in clozapine recipients by age group. All pvalues reported were two-sided, and the significance level was set at $\mathrm{p}<0.05$. Statistical analysis was performed using $\mathrm{R}$ version 3.6.2 (R Development Core Team, Vienna, Austria).

\section{RESULTS}

\section{Study population}

The total number of patients enrolled in the CPMS database was 9,635. Of these, 1,196 patients were enrolled in duplicate after being transferred to another hospital, so they were excluded after consolidating the data from the hospitals before and after. Of these 8,439 patients, 22 were excluded who had individually imported clozapine prior to CPMS enrollment. Forty-eight patients had participated in the clinical trial and were excluded. Sixty-nine patients who did not receive clozapine after CPMS enrollment were excluded. Finally, there were 37 patients with an initial dose other than $12.5 \mathrm{mg}$, who were excluded because it was outside the scope of the current protocol. As a result, the number of patients was 8,263 (Figure 1).

\section{Clinical background of the patients}

The clinical background of the patients is shown in Table 1 . The proportion of males was high (54.1\%), and the median age was 40 years. The patients were introduced to clozapine because $92.7 \%$ had a poor response to previously used anti-

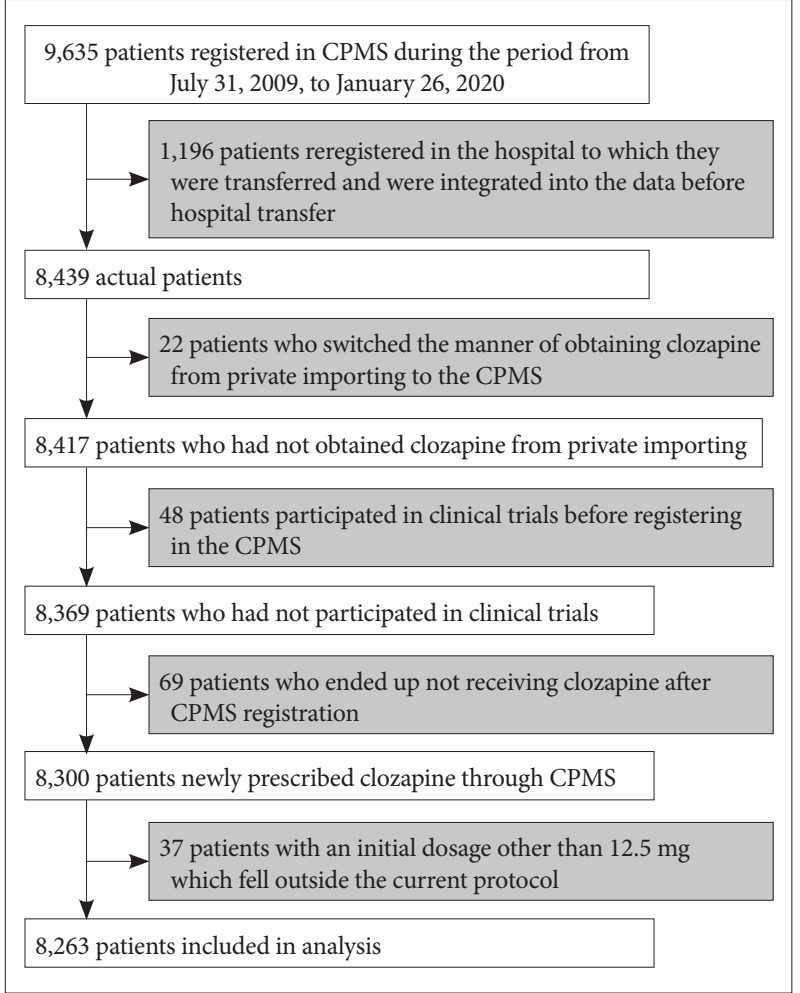

Figure 1. Flow diagram describing the construction of the study population. CPMS: Clozaril patient monitoring service ${ }^{\circledR}$.
Table 1. Clinical background of the patients at the registration with the Clozaril patient monitoring service ${ }^{\circledast}$

\begin{tabular}{lc}
\hline \multicolumn{2}{c}{ Background $(\mathrm{N}=8,263)$} \\
\hline Sex, $\mathrm{N}(\%)$ & $3,793(45.9)$ \\
Female & $4,470(54.1)$ \\
$\quad$ Male & $40(10-87)$ \\
Age, median (range), year* & \\
Age group, N (\%)* & $266(3.2)$ \\
$\leq 20$ & $1,457(17.6)$ \\
$21-30$ & $2,458(29.7)$ \\
$31-40$ & $2,273(27.5)$ \\
$41-50$ & $1,246(15.1)$ \\
$51-60$ & $494(6.0)$ \\
$61-70$ & $69(0.8)$ \\
$\geq 71$ &
\end{tabular}

Reason patient introduced clozapine use, N (\%)

Intolerance to previously used

antipsychotics $^{\dagger}$

$537(6.5)$

Aripiprazole $\ddagger$

$212(2.6)$

Blonanserin $^{\ddagger}$

$140(1.7)$

Olanzapine $^{\ddagger}$

$240(2.9)$

Paliperidone $e^{\ddagger}$

$83(1.0)$

Perospirone $\ddagger$

$40(0.5)$

Quetiapine $^{\ddagger}$

$91(1.1)$

Risperidone ${ }^{\ddagger}$

$257(3.1)$

Other SGA ${ }^{\ddagger}$

$45(0.5)$

Poor response to previously used

antipsychotics $^{\dagger}$

$7,659(92.7)$

Aripiprazole

$2,063(25.0)$

Blonanserin $^{\ddagger}$

$1,357(16.4)$

Olanzapine ${ }^{\ddagger}$

$4,650(56.3)$

Paliperidone ${ }^{\ddagger}$

$1,220(14.8)$

Perospirone $\ddagger$

$257(3.1)$

Quetiapine $^{\ddagger}$

$1,526(18.5)$

Risperidone $e^{\ddagger}$

$3,804(46.0)$

Other SGA ${ }^{\ddagger}$

304 (3.7)

FGA $^{\ddagger}$

$766(9.3)$

Previously treated with clozapine, N (\%)

Non-treated

$7,828(94.7)$

Treated

$435(5.3)$

Leukocyte, median (range), $/ \mathrm{mm}^{3}$

$6,000(4,000-20,500)$

Neutrophil, median (range), $/ \mathrm{mm}^{3}$

$3,397(2,000-16,572)$

Hemoglobin Alc, median (range), \%

$5.3(3.2-11.8)$

is expressed as the age at introduction of clozapine, tas some patients met neither or both of the requirements, the sum is not $100 \%$, fas all patients received more than two antipsychotics, the sum is more than the total. FGA: first-generation antipsychotics, SGA: second-generation antipsychotics 
psychotics, 6.5\% had intolerance to previously used antipsychotics, and $0.8 \%$ had an unknown reason. These antipsychotics are listed in descending order as follows: regarding previous poor responses, olanzapine (56.3\%), risperidone (46.0\%), aripiprazole (25.0\%), quetiapine (18.5\%), blonanserin (16.4\%), paliperidone (14.8\%), and perospirone (3.1\%), and regarding intolerance, risperidone $(3.1 \%)$, olanzapine $(2.9 \%)$, aripiprazole $(2.6 \%)$, blonanserin $(1.7 \%)$, quetiapine $(1.1 \%)$, paliperidone $(1.0 \%)$, and perospirone $(0.5 \%)$. There was $5.3 \%$ readministration of clozapine, i.e., previous treatment history with clozapine. The median leukocyte count, neutrophil count, and HbAlc were $6,000 / \mathrm{mm}^{3}, 3,397 / \mathrm{mm}^{3}$, and $5.3 \%$, respectively.

\section{Time to discontinuation of clozapine}

The Kaplan-Meier curves for clozapine discontinuation events are shown in Figures 2 and 3. Clozapine discontinuation occurred significantly earlier in the following groups: 40 years and older $(\mathrm{p}<0.001)$, intolerance to previously used antipsychotics ( $\mathrm{p}=0.009)$, no previous poor response to previously used antipsychotics $(\mathrm{p}<0.001)$, previous treatment with clozapine ( $\mathrm{p}<0.001)$, leukocytes less than $6,000 / \mathrm{mm}^{3}(\mathrm{p}<0.001)$, and neutrophils less than $3,397 / \mathrm{mm}^{3}(\mathrm{p}<0.001)$. No effects of sex $(p=0.597)$ and hemoglobin A1c $(p=0.071)$ were observed (Figure 2). Clozapine discontinuation occurred significantly earlier in patients with intolerance to former antipsychotics;
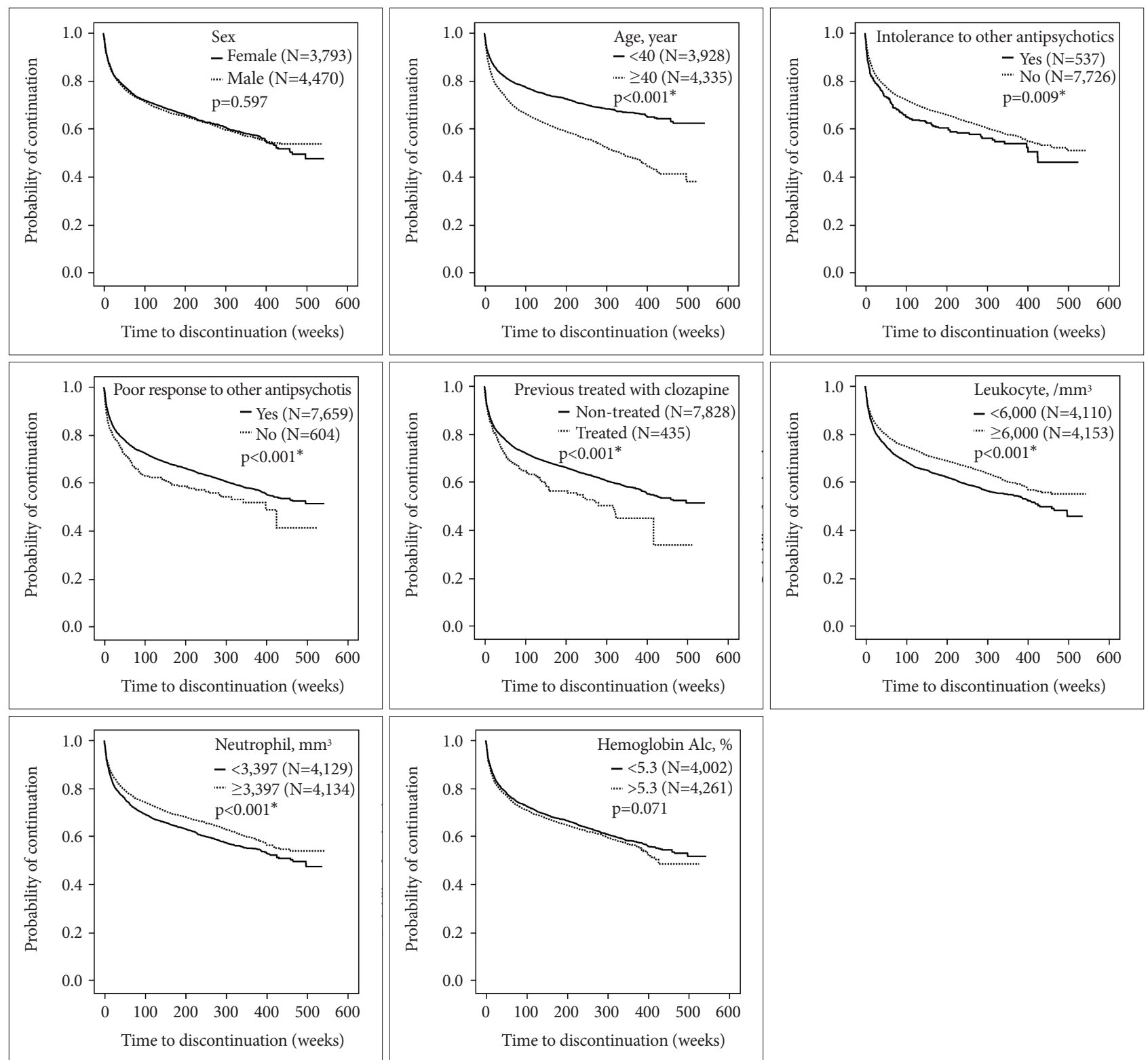

Figure 2. The Kaplan-Meier survival curves describe the time to discontinuation of clozapine by clinical background. The Kaplan-Meier eventfree curve was used to calculate the event-free probability of clozapine discontinuation. A log-rank test was performed to verify the differences between each group. ${ }^{*} p<0.05$. 
therefore, each antipsychotic was evaluated by the Kaplan-Meier curve, revealing that clozapine discontinuation occurred significantly earlier in patients with intolerance to olanzapine $(\mathrm{p}=0.007)$. No effects of intolerance to aripiprazole $(\mathrm{p}=0.113)$, blonanserin $(\mathrm{p}=0.426)$, paliperidone $(\mathrm{p}=0.509)$, perospirone $(\mathrm{p}=0.973)$, quetiapine $(\mathrm{p}=0.370)$, risperidone $(\mathrm{p}=0.058)$, and other second-generation antipsychotics $(p=0.513)$ were observed (Figure 3).

\section{Independent risk factors for clozapine discontinuation}

Seven variables with $\mathrm{p}<0.2$ in the log-rank test were includ- ed in the multivariate analysis. Multivariate Cox regression analysis revealed four independent risk factors for clozapine discontinuation (Figure 4). Clozapine discontinuation was significantly associated with age 40 and older, intolerance to olanzapine, previous treatment with clozapine, and leukocyte counts $<6,000 / \mathrm{mm}^{3}$. Since intolerance and poor response to previously used antipsychotics and leukocyte count and neutrophil count had multicollinear relationships, intolerance and leukocyte count were selected, respectively. Among the poorly tolerated antipsychotics, we used aripiprazole, olanzapine, and risperidone with $\mathrm{p}<0.2$. VIF was calculated as a measure of multicollinearity. It was found that each VIF did not exceed 10. The as-
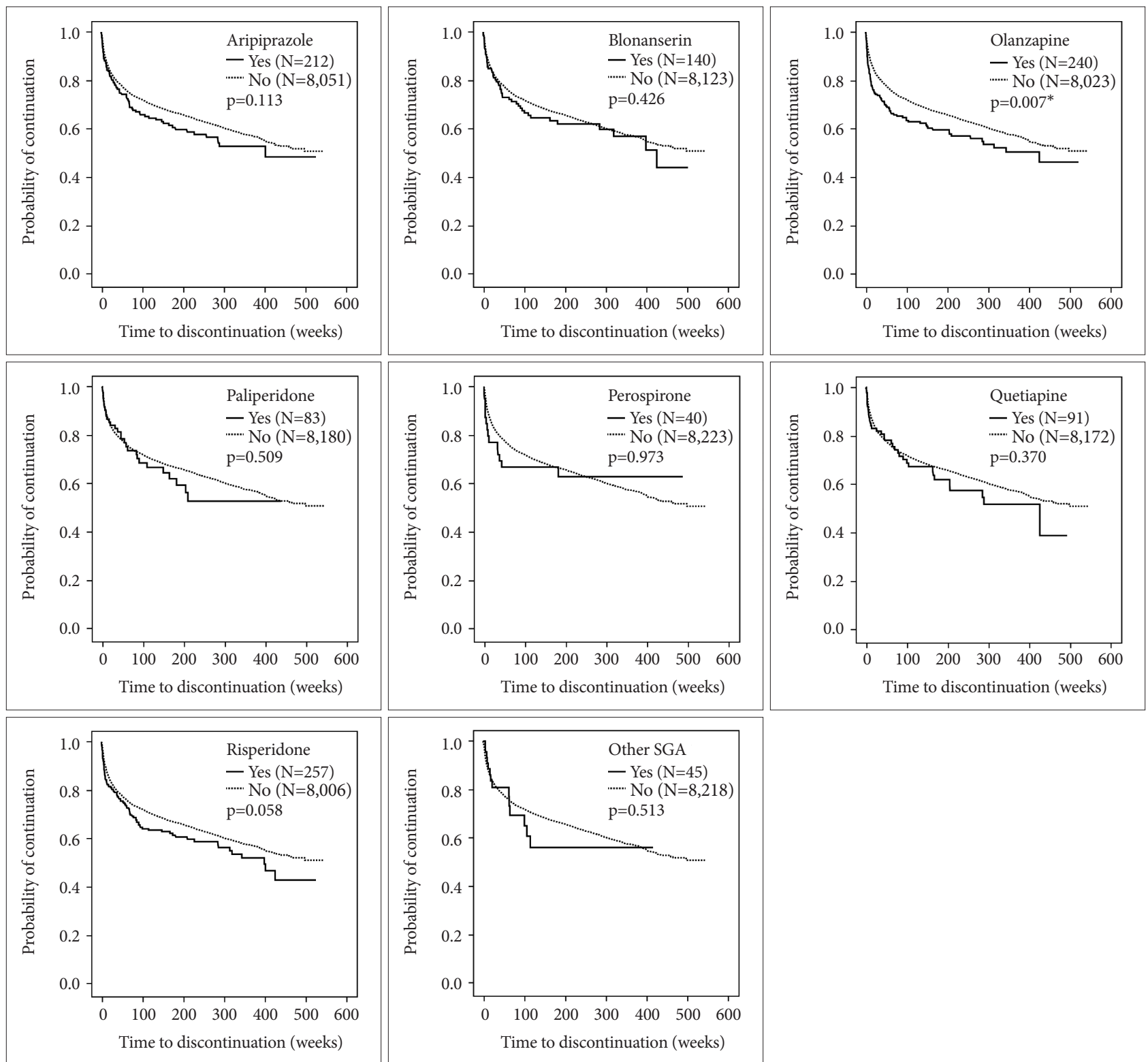

Figure 3. The Kaplan-Meier survival curves describe the time to discontinuation of clozapine based on intolerance to previously used antipsychotics. The Kaplan-Meier event-free curve was used to calculate the event-free probability of clozapine discontinuation. A log-rank test was performed to verify the differences between each group. ${ }^{*} p<0.05$. SGA: second-generation antipsychotics. 


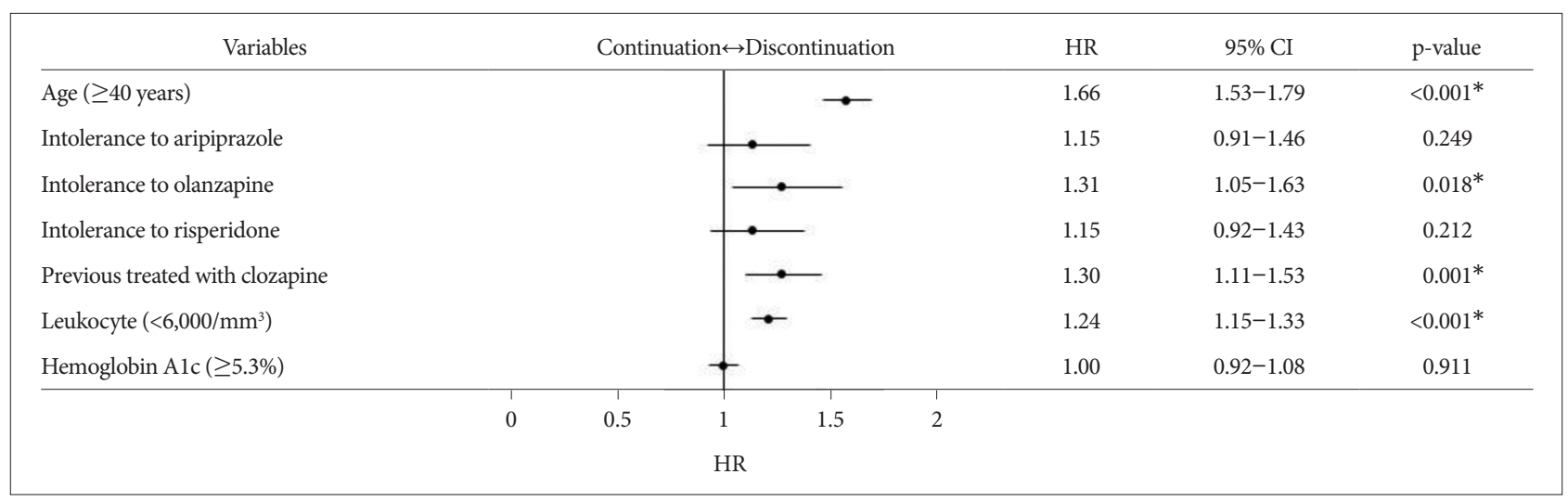

Figure 4. Multivariate Cox regression analysis for independent risk factors for clozapine discontinuation. Seven variables with $p<0.2$ in the log-rank test were included in the multivariate analysis. Multivariate Cox regression analysis was used to investigate independent risk factors associated with time to event. The event was the discontinuation of clozapine. VIFs of 10 or more were considered evidence of the existence of multicollinearity. It was found that each VIF did not exceed 10. The proportional hazards assumption was evaluated using the Kaplan-Meier curve. ${ }^{*} p<0.05$. Cl: confidence interval, HR: hazard ratio, VIF: variance inflation factor.

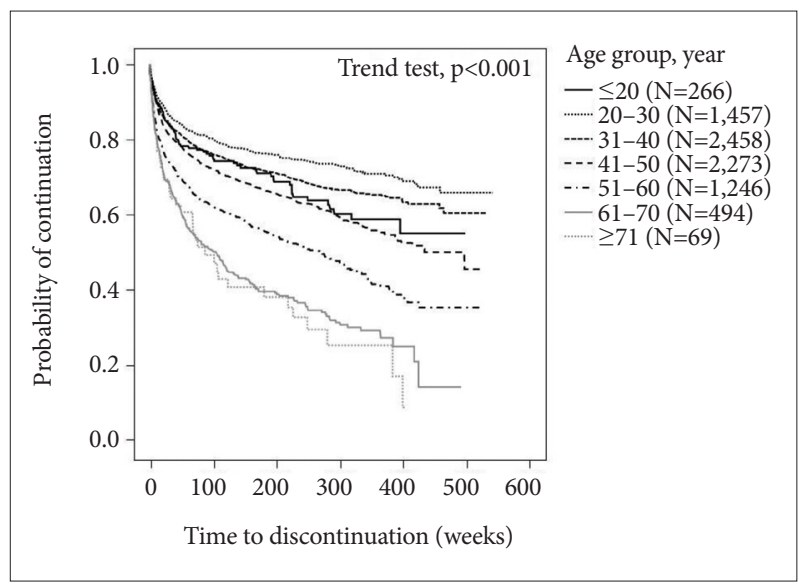

Figure 5. Trend of discontinuation rates of clozapine by age group. A trend test for survival data was performed for trends by age group in time to event. For the trend test, we assigned the median age within each level in the age group as the variable, that is, 18 for " $\leq 20$," 27 for " $21-30$," 36 for " $31-40$," 45 for " $41-50$," 55 for " $51-60$," 64 for "61-70," and 73 for " $\geq 71$." A Cox regression model was fitted to assess the trend of age group assigned these values.

sumption of proportional hazards was confirmed by KaplanMeier curves.

\section{Trend of discontinuation rates of clozapine by age group}

The Kaplan-Meier curves for clozapine discontinuation by age group revealed that older age at the time of clozapine introduction tended to have lower continuation rates (Figure 5). The 10-year continuation rate in each age group was $55.0 \%$ for “ $\leq 20$," $65.8 \%$ for " $21-30$ " (the highest continuation rate), $60.3 \%$ for “ $31-40$," $45.3 \%$ for “ $41-50$," $35.1 \%$ for “ $51-60$," and $13.7 \%$ for "61-70."

\section{Trend of incidence of hematopathy}

The incidence of agranulocytosis tended to be higher in old-

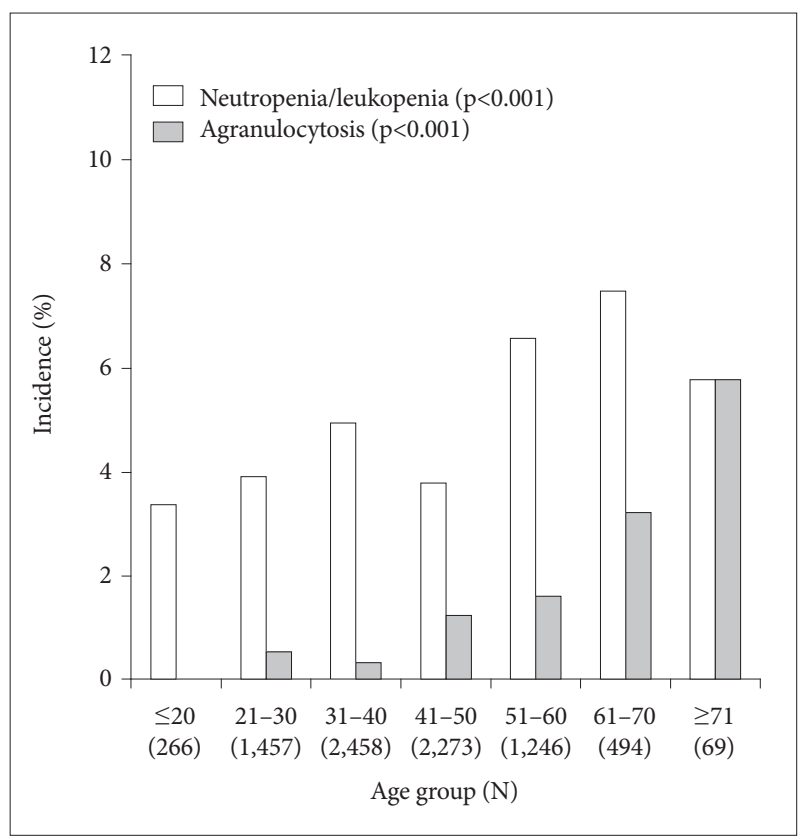

Figure 6. Trend of incidence of neutropenia/leukopenia and agranulocytosis in clozapine recipients by age group. The Cochran-Armitage trend test was performed to clarify the trend in the incidence of neutropenia/leukopenia and agranulocytosis in clozapine recipients by age group.

er age groups, although the incidence of neutropenia/leukopenia tended to be the similar increasing across ages (Figure 6).

\section{DISCUSSION}

This study is one of the nationwide surveys of clozapine prescription, with the largest sample size and nearly a single ethnicity. We focused on the risk factors for clozapine discontinuation and performed an analysis. The analysis revealed that being the group who was 40 years and older, who had poorly tolerated olanzapine, who had readministered clozapine, and 
with leukocyte counts $<6,000 / \mathrm{mm}^{3}$ were independent risk factors for clozapine discontinuation. Among these, the effect of older age had the highest risk of discontinuation, and other risk factors were similar. Furthermore, it was found that the rate of agranulocytosis increased significantly in patients aged 40 years and older, which probably contributed to the higher rate of clozapine discontinuation. Increasing the rate of agranulocytosis at higher age was consistent with studies conducted in the UK. ${ }^{17}$ However, in the UK study, the rate of neutropenia decreased with increases in age, but the current database showed an inconsistent result. Then, all the analyzed patients in the current study were of a high-risk ethnicity group, which may explain this inconsistency. Since the frequency of hematopathy differs between Japanese and Caucasians. Based on the previous study, ${ }^{17}$ Clozapine discontinuation due to agranulocytosis in Asian occurred 2.4 times as often as in Caucasians, so this Japanese result should be applied to other races with cautious. The causal genetic features of neutropenia and agranulocytosis caused by clozapine have already been identified from Japanese genetic data, ${ }^{18}$ and from now on, in addition to this result, it will be better to use genetic information and other biological data for the selection of clozapine usage, so the efficacy of clozapine will be enhanced.

Among the risk factors identified, the most influential risk factor was the age of clozapine introduction of 40 years or older. The result that the discontinuation rate of clozapine increased with the delay of the induction age was in agreement with the previous study. ${ }^{16}$ In addition, in a recent meta-analysis by Okhuijsen-Pfeifer et al., ${ }^{19}$ it is also consistent with the results of this study that relatively young age was an important predictor of better clozapine response. The age of 40 years old is considered to be the latest age of onset of schizophrenia, after which it is called late-onset schizophrenia. ${ }^{20}$ Generally, schizophrenia that develops from adolescence to early adulthood undergoes several recurrences and then transitions to the chronic stage around the age of $40 .^{21,22}$ It is controversial whether clozapine is effective against the cognitive impairment observed in the chronic phase. Studies with small sample sizes have reported an improvement by clozapine in some domains of cognitive function, ${ }^{23,24}$ however, it is not applicable to all domains. ${ }^{25}$ Although the current database did not include data on cognitive function, we are not able to conclude why 40 years old is the intersection between well- or poorly adapted patients. The present study found that continuation rates of clozapine in Japan varied by age of clozapine introduction. In other countries, there were reports that the continuation rate of clozapine in 24 months was $55 \%,{ }^{26}$ and in 400 days, it was approximately $80 \% ;{ }^{27}$ the average age of clozapine introduction in these studies was 36-37 years, which was somewhat younger than Japan. According to the Icelandic study, the mean age at introduction with clozap- ine was 37.8 years, i.e., less than 40 years old, with a 20 -year continuation rate of $71.2 \%{ }^{28}$ One of the main implications of the present study is that early introduction will contribute most to a high continuation rate of clozapine.

Since clozapine has a similar chemical skeleton to olanzapine, its pharmacological properties are also similar. It is known that side effects such as weight gain and blood glucose increases caused by olanzapine can also occur with clozapine. ${ }^{29}$ The clozapine continuation rate was significantly lower in patients who had intolerance to previously used antipsychotics (Figure 2). Only patients with intolerance to olanzapine had significantly lower clozapine continuation rates when assessed by individual antipsychotics (Figure 3). The clinical effects of clozapine observed in RCTs were higher than those of quetiapine or risperidone, but it was not significantly different from olanzapine. Furthermore, in the same study, clozapine was significantly longer in duration than quetiapine or risperidone, but the difference with olanzapine was not significant. ${ }^{30}$ On the other hand, there is a report that olanzapine was administered to 20 patients who experienced side effects such as agranulocytosis and convulsions due to clozapine, and clinical improvement in every patient was achieved without experiencing serious side effects. ${ }^{31}$ As suggested by these previous studies, olanzapine and clozapine show homology from clinical and pharmacological viewpoints, such as efficacy and tolerability, and olanzapine has been recognized as an alternative to clozapine. The present results are consistent with these findings. In the future, it is expected that the basis of characteristic side effects and clinical effects will be elucidated through the study of the pharmacological homology between the two drugs.

It was revealed that the clozapine readministration group was also a high-risk group with early discontinuation, although they represented a small group of 5.3\%. It is presumed that the readministration group had previously discontinued clozapine use due to some side effects or poor adherence. Clozapine rechallenge can be problematic and should be done with caution. In particular, in the case of interruption due to neutropenia or QT prolongation syndrome, it is recommended to increase the dose in small amounts. Based on the results of this study, rechallenge to patients with leukocyte counts $<6,000 / \mathrm{mm}^{3}$ should be done with caution. However, clozapine rechallenge is not recommended for patients with agranulocytosis or myocarditis, ${ }^{32}$ which leads to sudden death. Except for fatal side effects, the superiority of clozapine for treatment-resistant schizophrenia is established, and in fact, a recent meta-analysis revealed that clozapine has a significantly higher continuation rate than other second-generation antipsychotics (27\% reduction of discontinuation, NNT $=8, \mathrm{p}<0.001) .{ }^{33}$ Therefore, we would like to aim for more effective use by selecting an appropriate patient background and age based on the results of this study. 
This study has some inevitable limitations because this report is based solely on an analysis of CPMS data. First, the exact adherence of clozapine is unknown. Second, CPMS data use anonymous identification codes to protect the patient's personal information, so detailed information such as medical records, including age at onset, subclassification of schizophrenia, family history, symptomatic records scored by Positive and Negative symptoms scale (PANSS) and combination data related to drug metabolism or genetic data, are not included in the analysis. Third, there is a large variability in the number of individuals previously on other drugs, which can affect the pvalue of the analysis. Furthermore, no information is available on concomitant medications. For example, it is speculated that some doctors prescribe lithium for granulocyte depletion. Therefore, white blood cells and neutrophils are increased in patients receiving lithium, although it could not be considered adequately in this study because of the limitation of analyzed data source. One of the strengths of this study is that CPMS is a nationwide database of all cases in Japan and therefore directly reflects treatment with clozapine in one country. In addition, it can be said that off-label use of antipsychotics is not mixed because it is certain that the target patients for clozapine use are treatment-resistant schizophrenia patients.

In conclusion, we identified independent risk factors for clozapine discontinuation: aged 40 and older, intolerance to olanzapine, previous treatment with clozapine, and leukocyte counts $<6,000 / \mathrm{mm}^{3}$. Careful administration is important because patients with these factors have a high risk of discontinuation. In addition, a detailed examination of age revealed that introduction of clozapine during younger periods was more effective and better tolerated.

\section{Acknowledgments}

The authors thank the Japanese Society of Neuropsychopharmacology for their cooperation in data acquisition.

\section{Conflicts of Interest}

Dr. Toyoda has received speaker's honoraria from Eisai. Dr. Yamauchi has received speaker's honoraria from Meiji Seika Pharma, Otsuka. Dr. Kinoshita has received speaker's honoraria from Meiji Seika Pharma, Otsuka, Sumitomo Dainippon Pharma, EA Pharma and Eisai. Dr. Inada has received speaker's honoraria from Eisai, Eli Lilly, Janssen, Meiji Seika Pharma, Mitsubishi Tanabe Pharma, Mochida, MSD, Novartis, Otsuka, Shionogi, Sumitomo Dainippon Pharma, and Yoshitomiyakuhin, and research grants from Ministry of Health, Labor and Welfare, Mitsubishi Tanabe Pharma, MSD, and National Center of Neurology and Psychiatry. Dr. Kanazawa has received speaker's honoraria from Janssen, Meiji Seika Pharma, Otsuka, Shionogi, Sumitomo Dainippon Pharma, and Takeda Pharmaceutical Company, research grants from Ministry of Health, Labor and Welfare, and Health and Labor Sciences Research Grant (20GC1017 Head: Takefumi Ueno). The other authors declare no conflicts of interest.

\section{Author Contributions}

Conceptualization: Katsunori Toyoda, Takeo Hata, Ken Inada, Tetsufumi Kanazawa. Data curation: Katsunori Toyoda, Takeo Hata. Formal analysis:

Katsunori Toyoda, Takeo Hata, Tetsufumi Kanazawa. Funding acquisition: Tetsufumi Kanazawa. Investigation: Katsunori Toyoda, Takeo Hata, Tetsufumi Kanazawa. Methodology: Katsunori Toyoda, Takeo Hata. Project administration: Tetsufumi Kanazawa. Resources: Tetsufumi Kanazawa. Software: Takeo Hata. Supervision: Tetsufumi Kanazawa. Validation: Katsunori Toyoda, Takeo Hata, Tetsufumi Kanazawa. Visualization: Takeo Hata. Writingoriginal draft: Katsunori Toyoda. Writing_review \& editing: all authors.

\section{ORCID iDs}

Katsunori Toyoda

Takeo Hata

Shigeru Yamauchi

Shinya Kinoshita

Masami Nishihara

Kazuhisa Uchiyama

Ken Inada

Tetsufumi Kanazawa

https://orcid.org/0000-0002-7196-278X
https://orcid.org/0000-0001-5894-3552
https://orcid.org/0000-0002-3144-4128
https://orcid.org/0000-0002-2729-020X
https://orcid.org/0000-0001-7976-2750
https://orcid.org/0000-0003-3001-8722
https://orcid.org/0000-0002-3073-4588
https://orcid.org/0000-0003-3781-297X

\section{REFERENCES}

1. Chisholm D, Gureje O, Saldivia S, Villalón Calderón M, Wickremasinghe R, Mendis N, et al. Schizophrenia treatment in the developing world: an interregional and multinational cost-effectiveness analysis. Bull World Health Organ 2008;86:542-551.

2. Provencher HL, Mueser KT. Positive and negative symptom behaviors and caregiver burden in the relatives of persons with schizophrenia. Schizophr Res 1997;26:71-80.

3. Hawton K, Sutton L, Haw C, Sinclair J, Deeks JJ. Schizophrenia and suicide: systematic review of risk factors. Br J Psychiatry 2005;187:9-20.

4. Palmer CS, Revicki DA, Genduso LA, Hamilton SH, Brown RE. A costeffectiveness clinical decision analysis model for schizophrenia. Am J Manag Care 1998;4:345-355.

5. Howes OD, McCutcheon R, Agid O, de Bartolomeis A, van Beveren NJM, Birnbaum ML, et al. Treatment-resistant schizophrenia: Treatment Response and Resistance in Psychosis (TRRIP) working group consensus guidelines on diagnosis and terminology. Am J Psychiatry 2017;174:216229.

6. Meltzer HY. Treatment-resistant schizophrenia--the role of clozapine. Curr Med Res Opin 1997;14:1-20.

7. Taipale H, Tanskanen A, Mehtälä J, Vattulainen P, Correll CU, Tiihonen J. 20-year follow-up study of physical morbidity and mortality in relationship to antipsychotic treatment in a nationwide cohort of 62,250 patients with schizophrenia (FIN20). World Psychiatry 2020;19:61-68.

8. Nucifora FC, Mihaljevic M, Lee BJ, Sawa A. Clozapine as a model for antipsychotic development. Neurotherapeutics 2017;14:750-761.

9. Tiihonen J, Wahlbeck K, Lönnqvist J, Klaukka T, Ioannidis JPA, Volavka J, et al. Effectiveness of antipsychotic treatments in a nationwide cohort of patients in community care after first hospitalisation due to schizophrenia and schizoaffective disorder: observational follow-up study. BMJ 2006;333:224.

10. Essock SM, Frisman LK, Covell NH, Hargreaves WA. Cost-effectiveness of clozapine compared with conventional antipsychotic medication for patients in state hospitals. Arch Gen Psychiatry 2000;57:987994.

11. Bachmann CJ, Aagaard L, Bernardo M, Brandt L, Cartabia M, Clavenna $\mathrm{A}$, et al. International trends in clozapine use: a study in 17 countries. Acta Psychiatr Scand 2017;136:37-51.

12. Xu SW, Dong M, Zhang Q, Yang SY, Chen LY, Sim K, et al. Clozapine prescription pattern in patients with schizophrenia in Asia: The REAP survey (2016). Psychiatry Res 2020; 287:112271.

13. Whiskey E, Wykes T, Duncan-McConnell D, Haworth E, Walsh N, Hastilow S. Continuation of clozapine treatment: practice makes perfect. Psychiatr Bull 2003;27:211-213.

14. Knoph KN, Morgan RJ, Palmer BA, Schak KM, Owen AC, Leloux MR, et al. Clozapine-induced cardiomyopathy and myocarditis monitoring: 
a systematic review. Schizophr Res 2018;199:17-30.

15. Moeller FG, Chen YW, Steinberg JL, Petty F, Ripper GW, Shah N, et al. Risk factors for clozapine discontinuation among 805 patients in the VA hospital system. Ann Clin Psychiatry 1995;7:167-173.

16. Krivoy A, Malka L, Fischel T, Weizman A, Valevski A. Predictors of clozapine discontinuation in patients with schizophrenia. Int Clin Psychopharmacol 2011;26:311-315.

17. Munro J, O'Sullivan D, Andrews C, Arana A, Mortimer A, Kerwin R. Active monitoring of 12,760 clozapine recipients in the UK and Ireland. Beyond pharmacovigilance. Br J Psychiatry 1999;175:576-580.

18. Saito T, Ikeda M, Mushiroda T, Ozeki T, Kondo K, Shimasaki A, et al. Pharmacogenomic study of clozapine-induced agranulocytosis/granulocytopenia in a Japanese population. Biol Psychiatry 2016;80:636-642.

19. Okhuijsen-Pfeifer C, Sterk AY, Horn IM, Terstappen J, Kahn RS, Luykx JJ. Demographic and clinical features as predictors of clozapine response in patients with schizophrenia spectrum disorders: a systematic review and meta-analysis. Neurosci Biobehav Rev 2020;111:246-252.

20. Howard R, Rabins PV, Seeman MV, Jeste DV. Late-onset schizophrenia and very-late-onset schizophrenia-like psychosis: an international consensus. The International Late-Onset Schizophrenia Group. Am J Psychiatry 2000;157:172-178.

21. Lieberman JA, Perkins D, Belger A, Chakos M, Jarskog F, Boteva K, et al. The early stages of schizophrenia: speculations on pathogenesis, pathophysiology, and therapeutic approaches. Biol Psychiatry 2001;50: 884-897.

22. Lieberman JA. Neurobiology and the natural history of schizophrenia. J Clin Psychiatry 2006;67:e14.

23. Lee MA, Thompson PA, Meltzer HY. Effects of clozapine on cognitive function in schizophrenia. J Clin Psychiatry 1994;55(Suppl B):82-87.

24. McGurk SR. The effects of clozapine on cognitive functioning in schizophrenia. J Clin Psychiatry 1999;60(Suppl 1):24-29.

25. Goldberg TE, Weinberger DR. The effects of clozapine on neurocogni- tion: an overview. J Clin Psychiatry 1994;55(Suppl B):88-90.

26. Legge SE, Hamshere M, Hayes RD, Downs J, O’Donovan MC, Owen MJ, et al. Reasons for discontinuing clozapine: a cohort study of patients commencing treatment. Schizophr Res 2016;174:113-119.

27. Ascher-Svanum H, Zhu B, Faries D, Landbloom R, Swartz M, Swanson $\mathrm{J}$. Time to discontinuation of atypical versus typical antipsychotics in the naturalistic treatment of schizophrenia. BMC Psychiatry 2006;6:8.

28. Ingimarsson $\mathrm{O}, \mathrm{MacCabe} \mathrm{JH}$, Haraldsson M, Jónsdóttir $\mathrm{H}$, Sigurdsson E. Clozapine treatment and discontinuation in Iceland: a national longitudinal study using electronic patient records. Nord J Psychiatry 2016; 70:450-455.

29. Pillinger T, McCutcheon RA, Vano L, Mizuno Y, Arumuham A, Hindley $\mathrm{G}$, et al. Comparative effects of 18 antipsychotics on metabolic function in patients with schizophrenia, predictors of metabolic dysregulation, and association with psychopathology: a systematic review and network meta-analysis. Lancet Psychiatry 2020;7:64-77.

30. McEvoy JP, Lieberman JA, Stroup TS, Davis SM, Meltzer HY, Rosenheck RA, et al. Effectiveness of clozapine versus olanzapine, quetiapine, and risperidone in patients with chronic schizophrenia who did not respond to prior atypical antipsychotic treatment. Am J Psychiatry 2006; 163:600-610.

31. Littrell KH, Johnson CG, Hilligoss NM, Peabody CD, Littrell SH. Switching clozapine responders to olanzapine. J Clin Psychiatry 2000;61:912915.

32. Manu P, Lapitskaya Y, Shaikh A, Nielsen J. Clozapine rechallenge after major adverse effects: clinical guidelines based on 259 cases. Am J Ther 2018;25:e218-e223.

33. Masuda T, Misawa F, Takase M, Kane JM, Correll CU. Association with hospitalization and all-cause discontinuation among patients with schizophrenia on clozapine vs other oral second-generation antipsychotics: a systematic review and meta-analysis of cohort studies. JAMA Psychiatry 2019;76:1052-1062. 